\title{
Application of side-view imaging and real-time hydrogen measurement to the investigation of magnesium corrosion
}

DOI:

$10.5006 / 2299$

\section{Document Version}

Accepted author manuscript

Link to publication record in Manchester Research Explorer

\section{Citation for published version (APA):}

Curioni, M., Torrescano Alvarez, J., Yang, Y. F., \& Scenini, F. (2017). Application of side-view imaging and realtime hydrogen measurement to the investigation of magnesium corrosion. Corrosion, 73(5).

https://doi.org/10.5006/2299

\section{Published in:}

Corrosion

\section{Citing this paper}

Please note that where the full-text provided on Manchester Research Explorer is the Author Accepted Manuscript or Proof version this may differ from the final Published version. If citing, it is advised that you check and use the publisher's definitive version.

\section{General rights}

Copyright and moral rights for the publications made accessible in the Research Explorer are retained by the authors and/or other copyright owners and it is a condition of accessing publications that users recognise and abide by the legal requirements associated with these rights.

\section{Takedown policy}

If you believe that this document breaches copyright please refer to the University of Manchester's Takedown Procedures [http://man.ac.uk/04Y6Bo] or contact uml.scholarlycommunications@manchester.ac.uk providing relevant details, so we can investigate your claim.

\section{OPEN ACCESS}




\title{
Application of side-view imaging and real-time hydrogen measurement to the investigation of magnesium corrosion
}

\author{
M. Curioni, J.M. Torrescano-Alvarez, Y.F. Yang, F. Scenini
}

Corrosion and Protection Centre, School of Materials, University of Manchester, Manchester, M139PL, United Kingdom

\begin{abstract}
This work illustrates how side-view optical imaging, anodic potentiodynamic polarization and realtime hydrogen evolution measurements can be applied and complementary used to study the processes occurring on a corroding magnesium surface. Side-view imaging reveals that hydrogen evolution takes three different forms: i) large and stable bubbles on the uncorroded regions, ii) a stream of fine hydrogen bubbles at the corrosion front and iii) medium-sized bubbles behind the corrosion front. The observation suggests that the relatively large bubbles ahead and behind the corrosion front are associated with a purely cathodic reaction that provides the 'remote current'. This current, combined with the depassivating role of chloride ions, maintains the corrosion front active, generating regions where either the metal is locally directly exposed to the electrolyte or it is only covered by a poorly protective chloride-rich film. Regardless of the precise nature or morphology of the poorly protective (or absent) surface film at the corrosion front, additional hydrogen evolution occurs due to the large overpotential available. Overall, the anodic current associated with magnesium oxidation is the sum of the 'remote current', which is manifested with hydrogen evolution ahead and behind the corrosion front, and the 'local' current associated with hydrogen evolution at the corrosion front. From the electrical viewpoint, the corrosion front acts as a current amplifier where the current amplification effect can be measured directly by real-time gravimetric hydrogen measurement performed simultaneously with anodic potentiodynamic polarization.
\end{abstract}




\section{Introduction}

The study of magnesium corrosion by electrochemical methods is non-trivial due to the multiple processes occurring on the metal surface. Among the structural metals of practical interest, magnesium has the lowest electrochemical potential for oxidation (-2.37 V vs SHE), which is well below the electrochemical potential required for hydrogen evolution. In addition, the magnesium surface supports the formation of an oxide/hydroxide layer that is only partially protective in aqueous environments at near neutral $\mathrm{pH}^{1}$. As a consequence, in an aqueous electrolyte, the presence of the air formed oxide/hydroxide film is not sufficient to prevent corrosion initiation and propagation. Generally, corrosion initiates locally and propagates parallel to the metal surface faster than it propagates perpendicularly to the metal surface ${ }^{2-7}$. This process results in the progressive propagation of dark-grey/black regions, and only once the entire metal surface appears dark, corrosion might proceed locally in-depth.

During the first stages of corrosion, i.e. when the transition from silvery to dark occurs, numerous processes take place on the surface. Immediately after immersion, the surface film thickens due to the large potential difference between magnesium oxidation and hydrogen evolution which takes place on exposed impurities and intermetallics. This stage is generally associated with a relatively fast increase in corrosion potential due to the decrease in anodic activity that follows film thickening. Once the potential has increased sufficiently, the original film undergoes breakdown and corrosion proceeds locally. As soon as this occurs the potential becomes relatively steady and hydrogen streams composed by very fine bubbles are observed at the interface between the silvery and the dark areas ${ }^{8}$.

Upon anodic polarization, the hydrogen evolution rate increases, generating the so called 'negative difference effect' or 'superfluous hydrogen evolution', which is contrary to what electrochemical theory would predict ${ }^{1,4,5,9-12}$. Conversely, upon cathodic polarization, the hydrogen evolution rate behaves as expected by the theory, i.e. it increases with increasing cathodic polarization ${ }^{2}$.

The peculiar corrosion behaviour observed on magnesium has been the focus of numerous studies in recent years and several interpretations have been proposed. Historically, one of the possible interpretations was the generation of univalent magnesium during anodic polarization ${ }^{10,13,14}$. The generation of univalent magnesium could in principle explain this behaviour, since univalent magnesium ions could oxidize to divalent magnesium after detaching and generate hydrogen after ejection into the electrolyte. However, a number of recent experimental evidences strongly suggest that univalent magnesium is not responsible for the negative difference effect, and the process of 
hydrogen evolution is entirely cathodic in nature. Discussion of the arguments supporting this statement is not reported here for brevity, but can be found in the literature ${ }^{2,4-6,15-17}$.

Accepting that hydrogen evolution on magnesium is not the consequence of the generation of univalent magnesium, it is necessary to explain the unexpected increase in hydrogen evolution rate during anodic polarization. Several mechanisms, not necessarily contrasting each other, have been proposed to date to account for the phenomena observed. Such mechanisms can be in the first approximation be grouped in there categories, each broadly attributing the increase in hydrogen evolution during anodic polarization: i) an increase in exchange current for hydrogen evolution with increasing anodic polarization, the so called 'autocatalytic effect' (for example ref ${ }^{4}$ ), ii) an increase in cathodic activity with increasing anodic polarization due to change in nature of the corroded surface following enrichment of impurities or increased exposure of second phase material after corrosion (for example ref ${ }^{18}$ ) and iii) an increase in hydrogen evolution due to the rupture of the film separating the metal from the electrolyte during anodic polarization (for example ref ${ }^{19}$ ).

This work aims to illustrate how the results obtained from side-view real-time imaging of the corroding magnesium surface and gravimetric hydrogen collection measurements performed simultaneously with anodic polarization can advance understanding of the hydrogen evolution processes on corroding magnesium.

\section{Experimental}

\section{Materials}

The experiments presented in this work were performed on $99.95 \mathrm{wt} . \% \mathrm{Mg}$, obtained from a cast ingot. Magnesium samples were mounted in epoxy resin, then wet ground and mechanically polished to 4000 grit paper. Corrosion testing was performed in naturally aerated $3.5 \mathrm{wt} . \% \mathrm{NaCl}$ solution prepared from analytical grade reagents (99.5\% pure, Fisher Scientific). For electrochemical testing an Iviumstat potentiostat was connected to a typical three electrode cell consisting of the magnesium specimen (working electrode), a platinum foil (counter electrode) and a saturated calomel electrode (reference electrode). The scan rate for anodic polarization was $1 \mathrm{mV} \mathrm{s}^{-1}$. Image acquisition was performed by using a Maplin USB microscope. For real-time hydrogen measurement a Ohaus Galaxy 160D (resolution $0.1 \mathrm{mg}$ ) laboratory balance was used. Some of the data presented have been rearranged from Ref $^{2}$.

\section{Real-time hydrogen evolution measurement by gravimetric method}


A schematic and a photograph of the apparatus for the measurement of hydrogen evolution, are shown in Figure 1. The key feature of this setup is an electrode holder coupled with a hydrogencollecting container. The holder comprises a rigid rod that supports a magnesium electrode that had previously been connected to an electrical wire and embedded in transparent epoxy resin; with this setup it is possible expose a well-defined area to the test electrolyte. Above the electrode, a cylindrical container that is open at the bottom but closed at the top container is attached to the rigid rod. In this way, during immersion, the hydrogen gas generated from the corroding magnesium electrode is collected at the top of the cylinder. The assembled electrode holder is then placed on the plate of a conventional laboratory balance or hung on the measuring hook fitted under some models of balance. Details of the two arrangements are available refs ${ }^{2,20}$ and ${ }^{21}$, respectively.

When hydrogen evolves from the corroding surface, it accumulates in the collecting cylinder, thereby displacing some of the test electrolyte from the collecting cylinder. As a result, a buoyancy force acting on the cylinder and the rod is generated, and it is transmitted mechanically to the balance. The buoyancy force is equal to the volume of hydrogen generated multiplied by the density of the test electrolyte. Considering that $1 \mathrm{~mol}$ of hydrogen evolved at room temperature occupies 22.46 litres, if the density of the test electrolyte is $1 \mathrm{~kg} \mathrm{dm}^{-3}$ (pure water), the buoyancy force generated is $22.46 \mathrm{~kg}$. By using Faraday's law, it is possible to calculate that $1 \mathrm{~mA}$ of current producing hydrogen at $100 \%$ efficiency in pure water generates a change in weight of $126 \mu \mathrm{g} \mathrm{s}$ or $7.6 \mathrm{mg} \mathrm{min}^{-1}$, a weight change that can be readily detected by a laboratory scale. It is important to note that the above figure needs to be scaled with the density of the solution, which in the above case was assumed to be $1 \mathrm{~kg} \mathrm{dm}^{-3}$. A detailed discussion on the effects of temperature and pressure for this method has been presented by Fajardo et al. ${ }^{21}$ and it is not repeated in this manuscript. Experiments were repeated numerous times, with some minor variations in the measured values of currents prior, during and after polarization, but a common overall behaviour was found. The differences in the measured values were attributed to the time that is required to adjust the experimental setup after immersion of the electrode in the environment. As a result, on some samples the corrosion was slightly more advanced then on other when the data were being recorded. Other errors are associated with transient until a steady state was achieved, and this include the time needed to remove completely the air from the submersed cylinder and the time needed for the spring back of the electrical cables which were deformed when immersing the sample.

\section{Side-view of corroding magnesium electrodes}


In order to obtain side-view images of corroding magnesium, the specimens needed to be prepared following a specific procedure. In this work, magnesium cubes of approximately $1 \times 1 \times 1 \mathrm{~cm}^{3}$ (Figure 2a) were cut from the ingot and embedded in epoxy resin (Figure 2b). After polymerization, the embedded specimens were ground such as two adjacent faces of the magnesium cube were exposed (Figure 2c). Subsequently, a thin layer of transparent epoxy resin was placed in one of the two exposed faces and a microscope glass was placed on the epoxy resin, such as one edge of the microscope glass was aligned with the edge of the exposed magnesium surface (Figure $2 \mathrm{~d}$ ). After polymerization of the resin, the glass was ground by 2000 and 4000 grit emery paper, such that the thickness of the glass nearby the edge of the exposed magnesium was substantially reduced compared to the original glass thickness (Figure 2e). This step is essential to avoid light reflection and image distortion due to the edge of the microscope glass. As a last step, the exposed magnesium surface was gently re-polished to ensure planarity between magnesium surface and glass and to remove contamination from the previous steps. Once prepared, the specimens were immersed in a purpose built cell with flat and thin glass walls, which was filled with approximately $100 \mathrm{ml}$ of $3.5 \%$ $\mathrm{NaCl}$. Side view images were acquired by a USB microscope placed outside the cell. An additional USB microscope was placed above the cell to acquire synchronised top-view images. The experiments were repeated numerous times, with consistent qualitative behaviour observed. However, due to the nature of the experiment which required to place the microscopes at one location and waiting for the corrosion front to appear, the images obtained are of very variable quality. In fact, during side view imaging, the corrosion front did not necessarily proceed from side to side of the field of view but it proceed in and out of the focal plane of the microscope. In this work, only the best images are reported.

\section{Results and discussion}

\section{Optical imaging of magnesium corroding at the open circuit potential}

Figure 3 presents a time series of side-view images of the same region of a magnesium electrode at the free corrosion potential. From the images, and more clearly from direct visual observation and from recorded videos (unreported), it is evident that three types of hydrogen bubbles are generated during corrosion. Specifically: i) large and slow growing bubbles are generated from the very early stages on the silvery uncorroded regions, ii) very fine bubbles (hydrogen streams) are always revealed at the corrosion front and iii) medium sized bubbles generate behind the corrosion front. Although not directly evident from the images presented here, examination of video recordings enable to conclude unequivocally that the both the large and medium bubbles always originate from 
the same locations, whereas the stream of hydrogen bubbles advances towards the uncorroded regions in a relatively regular manner.

From the side-view images, another interesting observation can be made (Figure 4,5). Specifically, it appears that most of the medium size bubbles, generated behind the corrosion front, rapidly grow and detach from the surface. However, it is not infrequent to observe bubbles that are detached from the surface but stationary, i.e. they don't float towards the surface. This is exemplified in Figure 5 , where a time sequence of the side-view of the corroded region is presented. The images are temporally spaced by $1 \mathrm{~s}$ and the red arrow indicates one bubble that is detached from the surface but that is stationary for the $5 \mathrm{~s}$ of duration of the image series. It is evident that other bubbles form and detach rapidly from the metal surface, but the bubble identified is present in all the images. It is worth mentioning that the simultaneous imaging from top and side-view (figure 4 and 5 ) indicates clearly that the stationary bubbles are not attached to the cell glass. Thus, it is possible that such stationary bubbles are trapped in a relatively thick layer covering the dark magnesium surface and consisting of precipitating magnesium hydroxide in the form of gel. Within this layer, the $\mathrm{pH}$ is expected to be substantially higher than above the uncorroded regions and the presence of such high-pH gel layer could explain the observation that corrosion mainly proceeds horizontally until all the silvery regions are attacked. The formation of gels during magnesium corrosion was observed also by other Authors. For example, Kirkland et al. ${ }^{6}$ investigated by optical imaging the dissolution behaviour of wedge-shaped magnesium specimens during galvanostatic anodic polarization, clearly revealing the formation of gel-like corrosion products. Williams et al. also suggested that the formation of gels plays a significant role in the corrosion behaviour of AZ31 alloy ${ }^{22}$ and gels were observed by Hiromoto et al. during experiments performed on pure magnesium in borate buffer solutions ${ }^{23}$.

Overall, the observations from the side-view imaging can be rationalized into the schematic presented in Figure 6, similarly to what has been suggested previously ${ }^{19}$. The large bubbles on the uncorroded regions generate from impurities that are directly exposed to the test electrolyte. Here, due to the low macroscopic potential of the magnesium electrode, cathodic hydrogen evolution takes place, producing alkalinisation which prevents significant attack of the surrounding material. Such cathodic process provides some of the total current that maintain active the corrosion front. A similar cathodic process occurs behind the corrosion front, where relatively large bubbles are always generated from the same locations. These locations are likely to be cathodically active since either the corrosion product film is less protective compared to the air-formed film, or intermetallics particles, or region with enrichment of impurities, have been exposed to the electrolyte after 
selective dissolution of the magnesium matrix. Similarly to the bubbles generated on the uncorroded regions, the cathodic process of hydrogen evolution provides the remaining part of the current that maintains active the corrosion front. At the corrosion front, the combined presence of the current generated by the previous two processes ('remotely') and of depassivating conditions such as chlorides or low $\mathrm{pH}$, results in rupture of the original surface film. Here, either the magnesium metal is directly exposed to the electrolyte, or a poorly-protective chloride-rich film is generated. Regardless the exact nature of the non passivated surface, the oxidation of magnesium and the hydrogen evolution occur simultaneously in these locations and as a consequence of the large potential difference between magnesium oxidation and hydrogen evolution. Thus, the current associated to magnesium oxidation is the sum of the 'remote current' due to hydrogen evolution ahead and behind the corrosion front and the 'local' current associated to hydrogen evolution at the corrosion front. From the electrical viewpoint, this induces a current amplification effect, since the current associated with magnesium oxidation is larger than the current produced 'remotely' by the larger bubbles ahead and behind the corrosion front, due to the local process of hydrogen evolution at the corrosion front. At this location, due to the unstable nature of the dissolving surface, the hydrogen bubbles cannot anchor and they detach almost immediately thus forming an hydrogen stream. As the corrosion front advances, a significant $\mathrm{pH}$ gradient is generated, with the $\mathrm{pH}$ behind the corrosion front being much higher than the $\mathrm{pH}$ ahead of the corrosion front. Such increase in $\mathrm{pH}$ might induce the formation of gels, whose viscous nature occasionally prevent some hydrogen bubble to float immediately.

\section{Hydrogen evolution measurements at the open circuit potential and during potentiodynamic polarization}

In order to quantify the current amplification effect, direct measurements of evolving hydrogen were performed together with potentiodynamic anodic polarization. Figure 7 presents the typical results that can be obtained by performing such an experiment. In Figure 7a, the potential-time behaviour is presented. After an initial $900 \mathrm{~s}$ period of free corrosion, the potential was linearly increased and the associated electrical current response is presented in Figure 7b. Figure 7c presents the change in weight as a function of time recorded during the experiment (electrode area $1.9 \mathrm{~cm}^{2}$ ), and in Figure 7d, the time evolution of the current calculated from hydrogen evolution is presented. It is evident that the current associated with hydrogen evolution follows closely the electrical current and that, when the application of electrical current terminates, the current associated with hydrogen evolution sharply decrease to a value that is comparable to what measured prior to polarization. 
Figure 8 presents the comparison between the electrical current and the hydrogen current as a function of time and applied potential. In the figure, the time on the horizontal axis enables comparison of the hydrogen current before, during and after anodic polarization. The value of potential during the anodic polarization is reported as a second horizontal axis. During the initial free corrosion period the current density calculated from the hydrogen evolved was in the region of 0.3 $\mathrm{mA} \mathrm{cm}{ }^{-2}$, but the signal was relatively noisy since such value is close to the detection limit of the setup for a specimen area of circa $2 \mathrm{~cm}^{-2}$. When the polarization was initiated at $900 \mathrm{~s}$, the electrical current rapidly increased. The current associated with hydrogen evolution also increased rapidly and the behaviour measured was similar to that of the electrical current. When the anodic polarization was terminated, the current associated to hydrogen evolution decreased rapidly to a value in the region of $1 \mathrm{~mA} \mathrm{~cm}^{-2}$, and higher than prior to polarization, but small compared to the value measured during polarization. This indicates that, in agreement with previous suggestions ${ }^{24,25}$, the increase in cathodic activity of the corroded regions during anodic polarization provides a minor contribution to the increase in hydrogen evolution rate during anodic polarization, and it is far from being the main controlling process. Considering the result in the light of the schematic of Figure 6 , it appears that, during anodic polarization, the remote current generated by purely cathodic regions on the specimen surface (large and medium bubbles), increases but such increase in current is very small compared to the remote current that is provided by the external circuit.

Under this assumption, the current amplification effect at the corrosion front can be quantified directly, as graphically illustrated in Figure 9. Here the various components of currents graphically represented by arrows in Figure 6 are plotted against the remote current, assumed, during an anodic polarization experiment, to be equal to the current applied by the potentiostat. From the figure, it is evident that the current that results in the oxidation of magnesium, which is given by the sum of the electrical current and the hydrogen current, increases approximately linearly with the remote current.

It is possible that the current amplification effect is responsible for the abrupt increase in corrosion rate with impurities level that is well documented for magnesium. If the impurity level is below a threshold limit, the remote current that is available at the free corrosion potential is insufficient to maintain an active corrosion front, and corrosion propagation is extremely low. If the level of impurities is sufficient, then the remote current produced is sufficient to maintain at least one stable active corrosion front. As the active corrosion front progresses parallel to the surface, it leaves behind corroded regions that are more cathodically active. This provides an additional remote current, which extends the active corrosion front and ultimately will produce more cathodically 
active regions. This suggestion is indirectly supported by the observations of Samaniego et al. ${ }^{24}$ and Fajardo et al. ${ }^{25}$, who reported that during anodic polarization (i.e. when the remote current provided by the external circuit largely exceeds that generated on the electrode surface), the increase in hydrogen evolution only shows a very weak dependence on the impurities level.

\section{Conclusions}

Side-view imaging, together with gravimetric hydrogen measurement during anodic polarization, provide effective tools to advance mechanistic understanding of magnesium corrosion. From the images of the corroding surface it appears that the hydrogen evolved ahead and behind the corrosion front is associated with a purely cathodic process that produces the current necessary to maintain an active corrosion front. At the corrosion front, the surface film is absent, or replaced by a poorly protective chloride-rich film; therefore, due to the large overpotential available, and the fact that hydrogen bubbles cannot anchor to the unstable nature of the dissolving surface, additional hydrogen evolution takes place in the form of streams. The overall effect is that the current produced remotely, ahead and behind the corrosion front is amplified at the corrosion front by the local hydrogen evolution. During anodic polarization, the increase in cathodic activity associated with the propagation of the dark corroded regions only provides a minor contribution to the overall increase in hydrogen evolving from the surface and the current amplification effect can be quantified.

Acknowledgments. EPSRC is acknowledged for provision of financial support through the LATEST2 Programme Grant. The CONACYT Programme is also acknowledged for financially supporting J.M.T. 


\section{Bibliography}

1. G.L. Song, A. Atrens, Advanced Engineering Materials 1, 1 (1999): p. 11-33.

2. M. Curioni, Electrochimica Acta 120, (2014): p. 284-292.

3. J. Janiec-Anwar, G. Thompson, X.R. Zhou, M. Curioni, M. Turski, T. Wilks, in Galvanic Corrosion between Magnesium Alloys and Steel, ed., vol. 765 2013), p. 648-652.

4. $\quad$ G.S. Frankel, A. Samaniego, N. Birbilis, Corrosion Science 70, (2013): p. 104-111.

5. G. Williams, N. Birbilis, H.N. McMurray, Electrochemistry Communications 36, (2013): p. 15.

6. N.T. Kirkland, G. Williams, N. Birbilis, Corrosion Science 65, (2012): p. 5-9.

7. A.D. King, N. Birbilis, J.R. Scully, Electrochimica Acta 121, (2014): p. 394-406.

8. L. Yang, X. Zhou, M. Curioni, S. Pawar, H. Liu, Z. Fan, G. Scamans, G. Thompson, Journal of the Electrochemical Society 162, 7 (2015): p. C362-C368.

9. G. Song, A. Atrens, Advanced Engineering Materials 5, 12 (2003): p. 837-858.

10. G. Song, A. Atrens, D. St. John, X. Wu, J. Nairn, Corrosion Science 39, 10-11 (1997): p. 19812004.

11. G. Song, A. Atrens, D. Stjohn, J. Nairn, Y. Li, Corrosion Science 39, 5 (1997): p. 855-875.

12. S. Thomas, N.V. Medhekar, G.S. Frankel, N. Birbilis, Curr. Opin. Solid State Mat. Sci., (0000):

13. R.L. Petty, A.W. Davidson, J. Kleinberg, Journal of the American Chemical Society 76, 2 (1954): p. 363-366.

14. T.R. Thomaz, C.R. Weber, T. Pelegrini, L.F.P. Dick, G. Knörnschild, Corrosion Science 52, 7 (2010): p. 2235-2243.

15. G. Williams, H. Neil McMurray, Journal of the Electrochemical Society 155, 7 (2008): p. C340-C349.

16. S. Lebouil, A. Duboin, F. Monti, P. Tabeling, P. Volovitch, K. Ogle, Electrochimica Acta, (2013):

17. V. Shkirskiy, A.D. King, O. Gharbi, P. Volovitch, J.R. Scully, K. Ogle, N. Birbilis, ChemPhysChem 16, 3 (2015): p. 536-539.

18. M. Taheri, J.R. Kish, N. Birbilis, M. Danaie, E.A. McNally, J.R. McDermid, Electrochimica Acta 116, (2014): p. 396-403.

19. Y. Yang, F. Scenini, M. Curioni, Electrochimica Acta 198, (2016): p. 174-184.

20. M. Curioni, F. Scenini, T. Monetta, F. Bellucci, Electrochimica Acta 166, 0 (2015): p. 372-384.

21. S. Fajardo, G.S. Frankel, Journal of the Electrochemical Society 162, 14 (2015): p. C693-C701.

22. G. Williams, R. Grace, R.M. Woods, Corrosion 71, 2 (2015): p. 184-198.

23. S. Hiromoto, A. Yamamoto, N. Maruyama, H. Somekawa, T. Mukai, Corrosion Science 50, 12 (2008): p. 3561-3568.

24. A. Samaniego, N. Birbilis, X. Xia, G.S. Frankel, Corrosion 71, 2 (2014): p. 224-233.

25. S. Fajardo, G.S. Frankel, Electrochimica Acta 165, (2015): p. 255-267. 


\section{Figure Captions}

Figure 1. Schematic representation (a) and photographic image (b) of the electrode holder with hydrogen collecting cylinder.

Figure 2. Schematic representation of the specimen preparation procedure to enable side-view imaging of the corrosion processes.

Figure 3. Time series of side-view images of the corroding magnesium electrode. In the top image the three different types of hydrogen evolution are labelled, specifically i) large and slow growing bubbles forming on the silvery uncorroded regions, ii) very fine bubbles (hydrogen streams) at the corrosion front and iii) medium sized bubbles generate behind the corrosion front. The time reported on the images is measured from acquisition of the top image, and it does not correspond to the total immersion time.

Figure 4: Top view images of a corroding magnesium surface. The dashed rectangle indicates the region corresponding to the side-view images of Figure 5. This was acquired simultaneously to the image labelled 3s in Figure 5.

Figure 5. Time sequence of the side-view images of the region corresponding to the dashed rectangle in figure 4 . The red arrow in each image indicates one bubble that is detached from the surface but does not float immediately. The time reported on the images is measured from acquisition of the top-left image, and it does not correspond to the total immersion time.

Figure 6. Schematic illustration of the processes taking place on the corroding magnesium surface.

Figure 7. Time evolution of a) the potential, b) the electrical current density, c) the weight difference due to collection of the evolved hydrogen and d) current density associated to the evolved hydrogen.

Figure 8. Comparison between the electrical current and the current associated to hydrogen evolution as a function of time and applied potential.

Figure 9. Dependency of the current producing magnesium oxidation on the value of remote current, under the assumption that during anodic polarization the external current largely exceeds the remote cathodic current generated on the electrode surface (ref. Figure 6). 

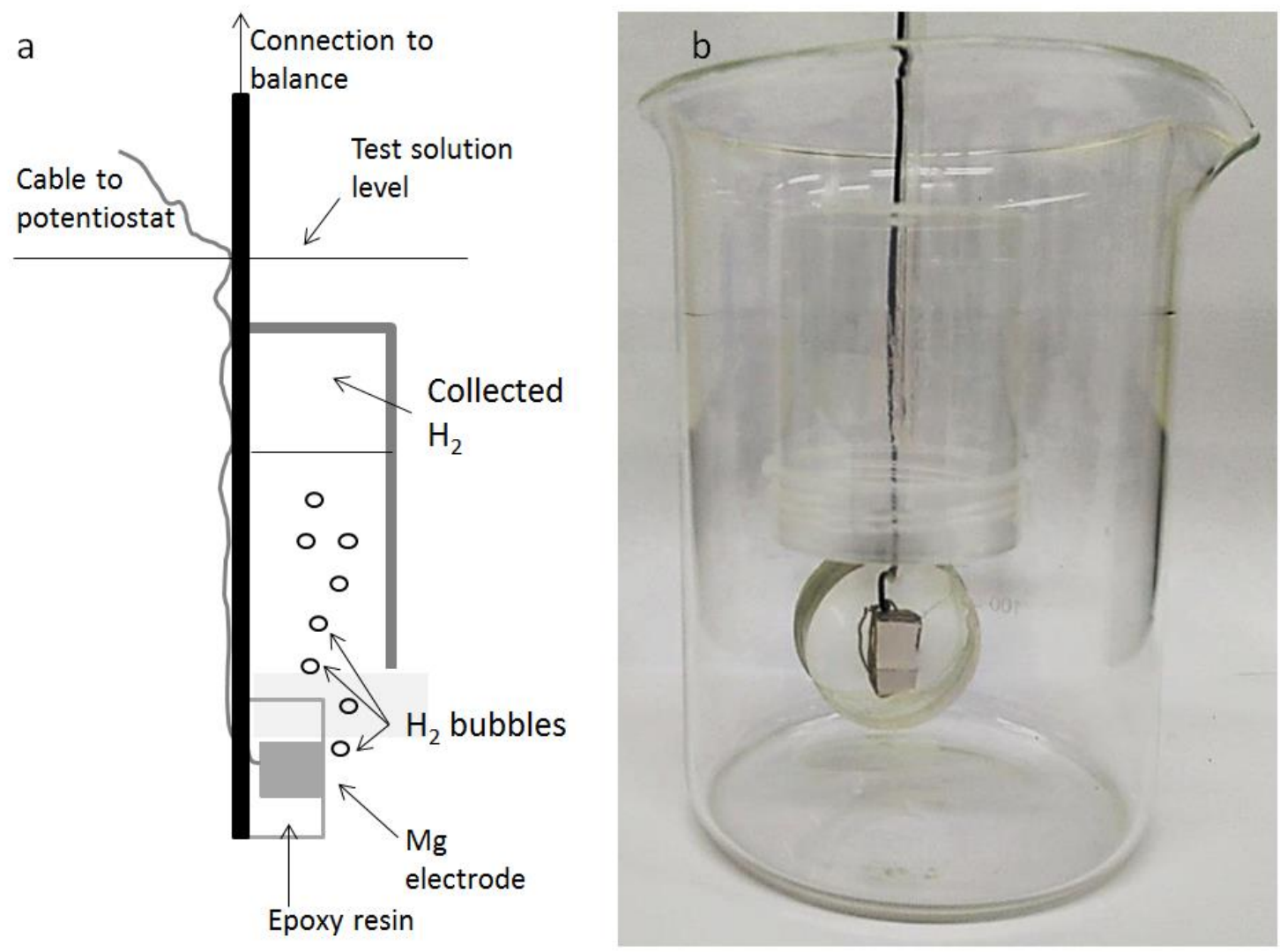

Figure 1. Schematic representation (a) and photographic image (b) of the electrode holder with hydrogen collecting cylinder. 

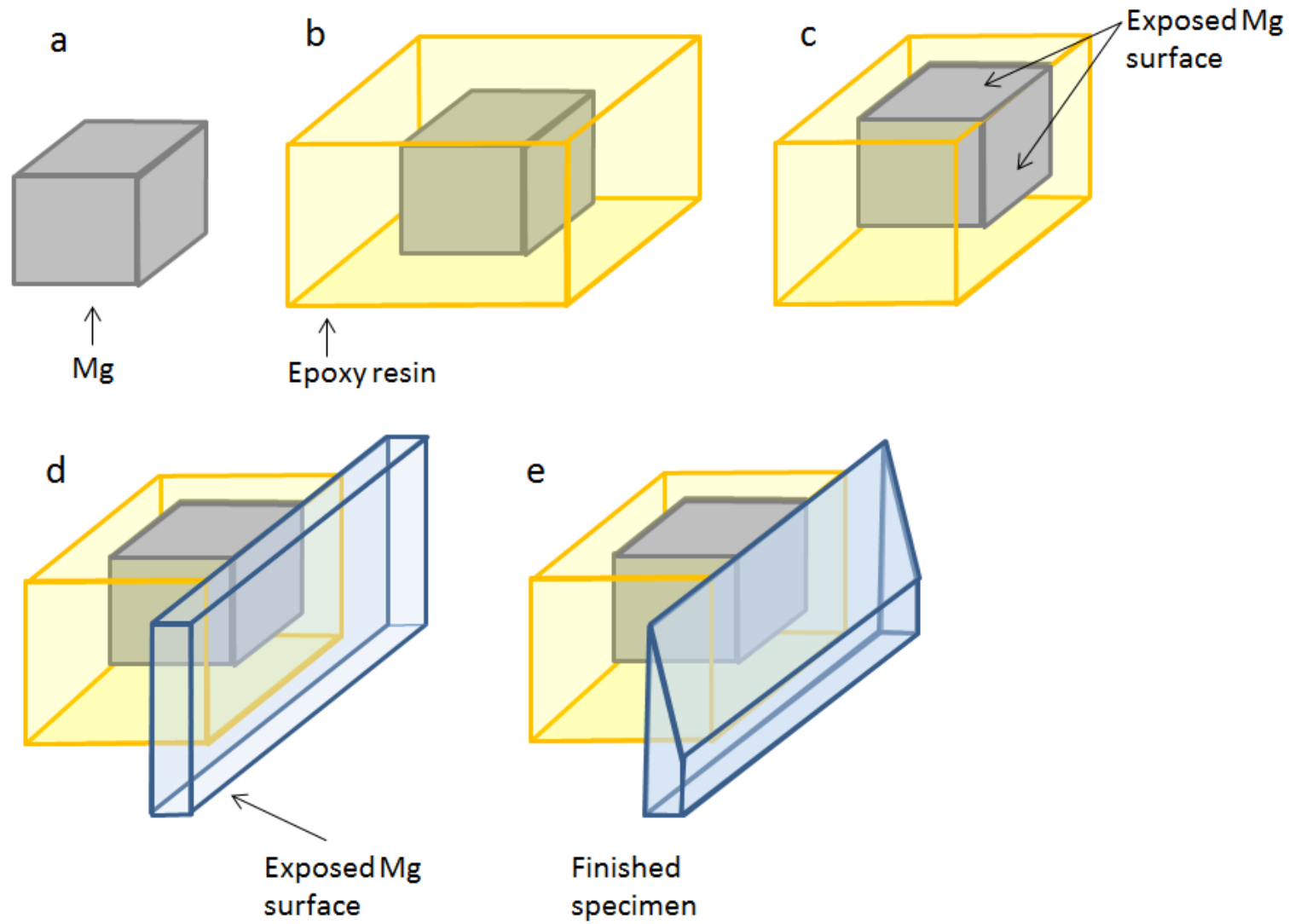

Figure 2. Schematic representation of the specimen preparation procedure to enable side-view imaging of the corrosion processes. 

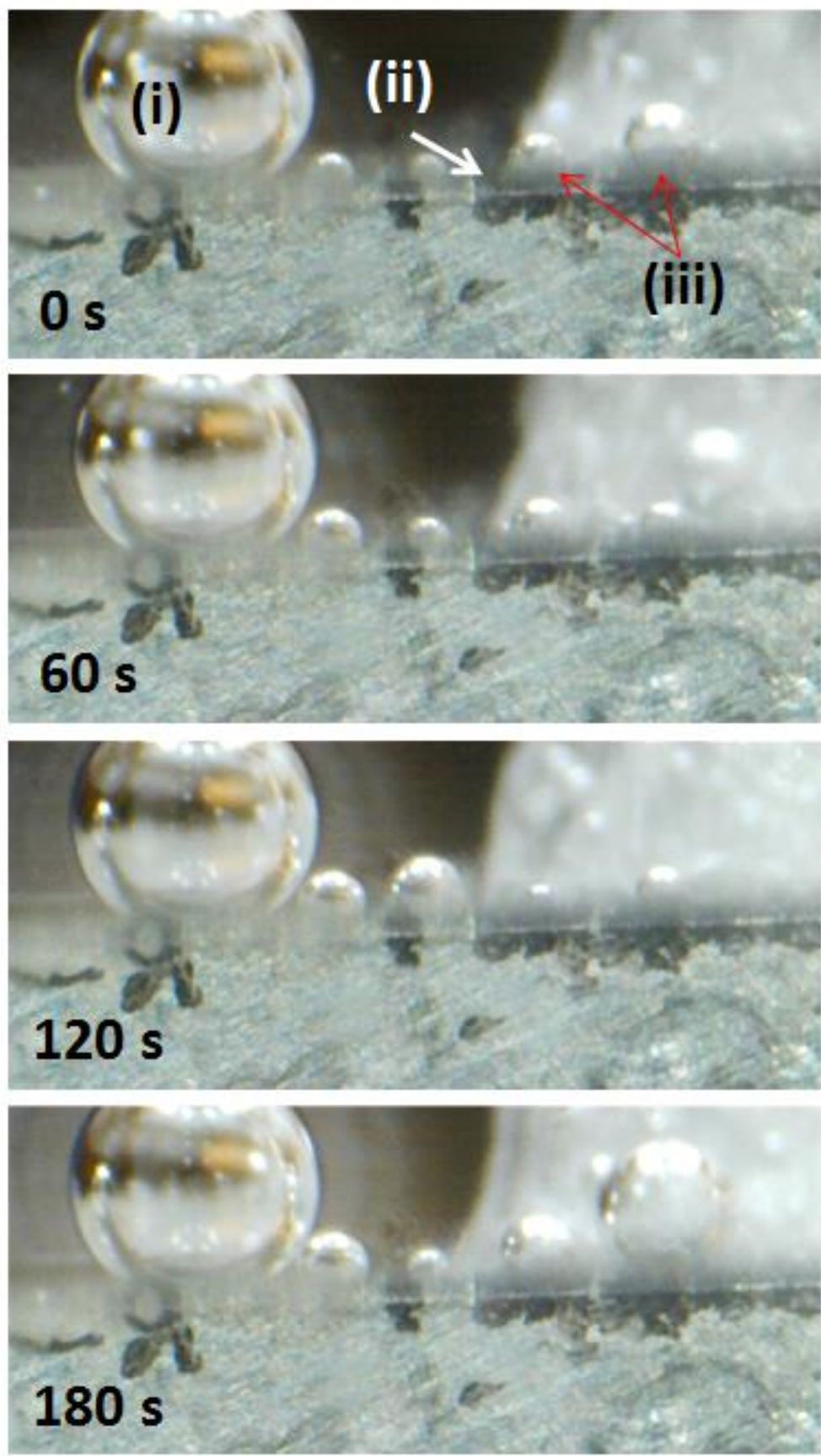

Figure 3. Time series of side-view images of the corroding magnesium electrode. In the top image the three different types of hydrogen evolution are labelled, specifically i) large and slow growing bubbles forming on the silvery uncorroded regions, ii) very fine bubbles (hydrogen streams) at the corrosion front and iii) medium sized bubbles generate behind the corrosion front. The time reported on the images is measured from acquisition of the top image, and it does not correspond to the total immersion time. 


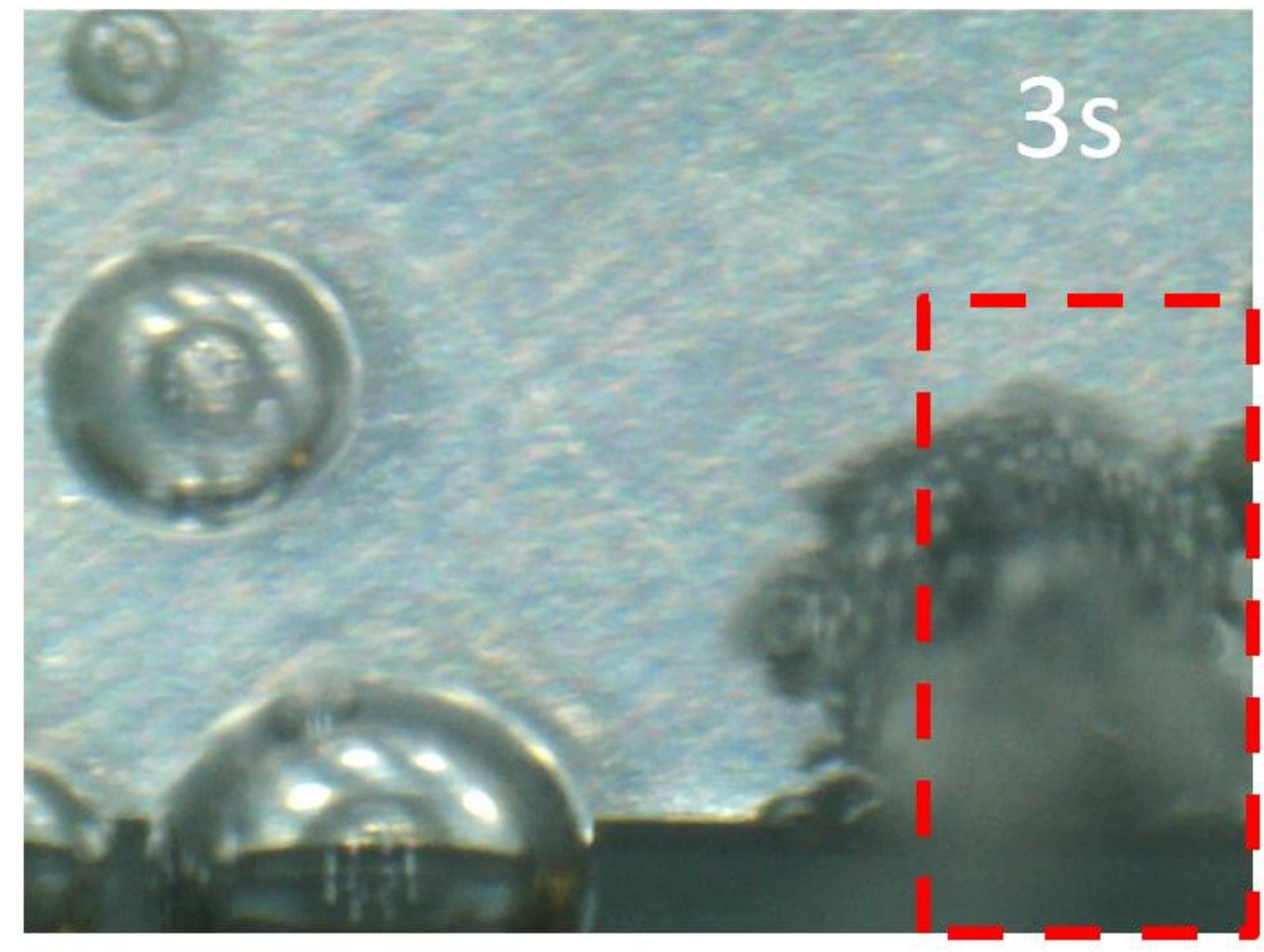

Figure 4: Top view images of a corroding magnesium surface. The dashed rectangle indicates the region corresponding to the side-view images of Figure 5. This was acquired simultaneously to the image labelled 3s in Figure 5. 

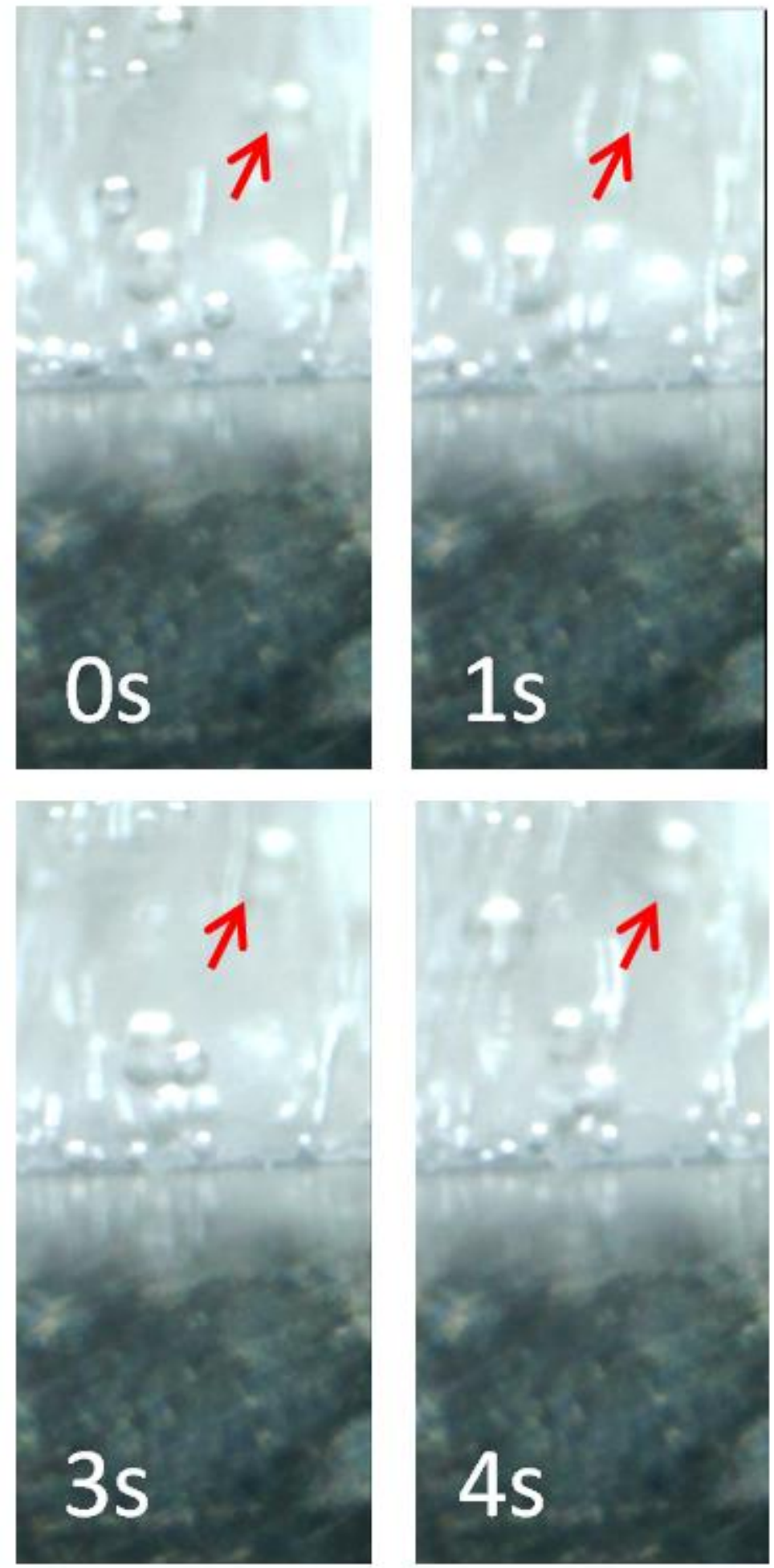
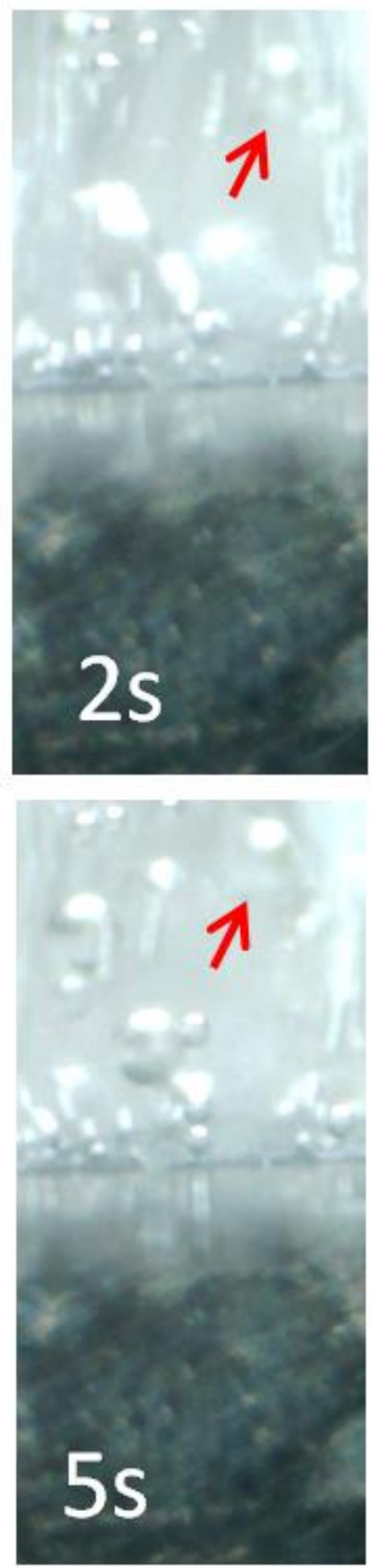

Figure 5. Time sequence of the side-view images of the region corresponding to the dashed rectangle in figure 4 . The red arrow in each image indicates one bubble that is detached from the surface but does not float immediately. The time reported on the images is measured from acquisition of the top-left image, and it does not correspond to the total immersion time. 


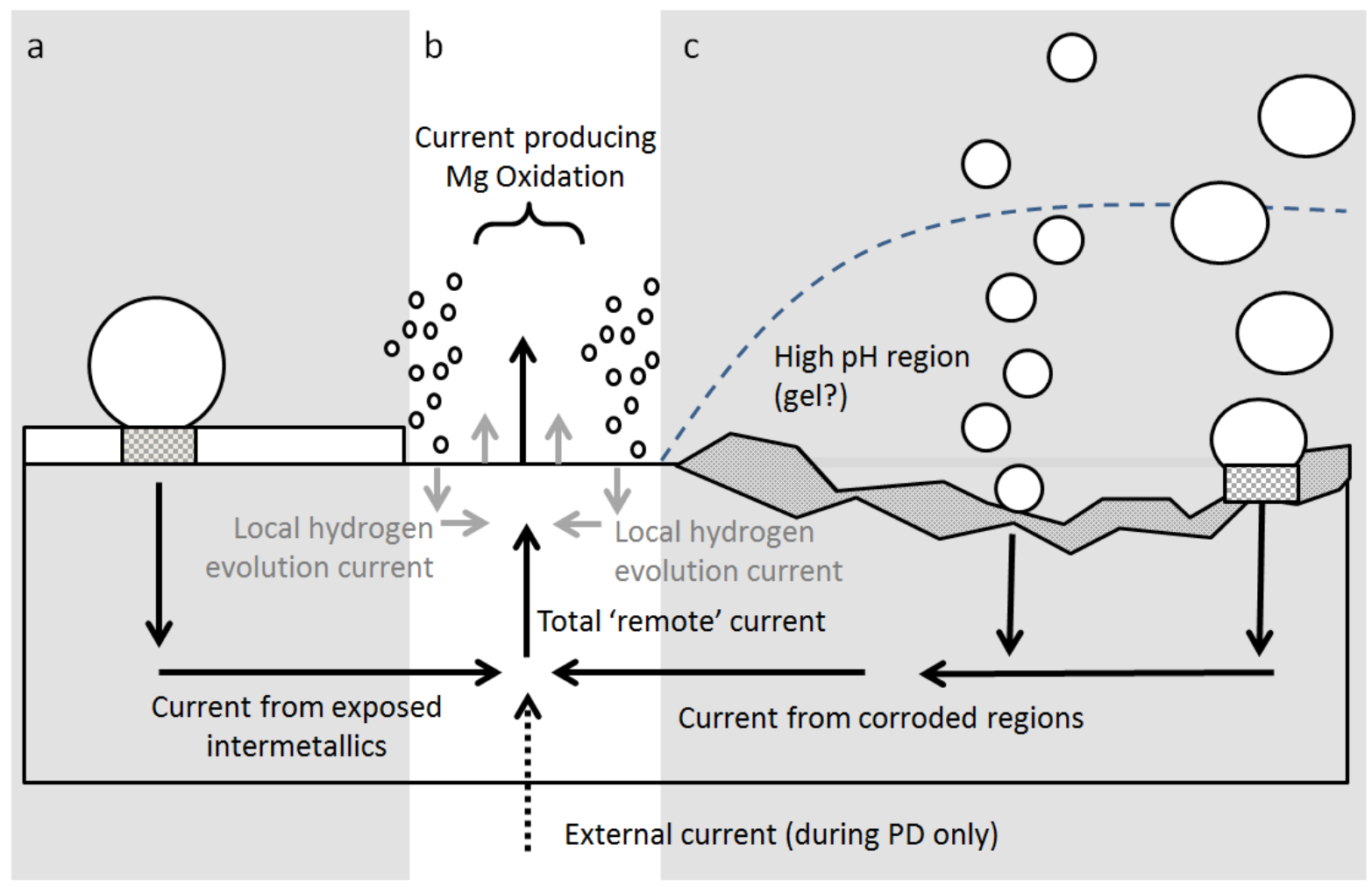

Figure 6. Schematic illustration of the processes taking place on the corroding magnesium surface. 


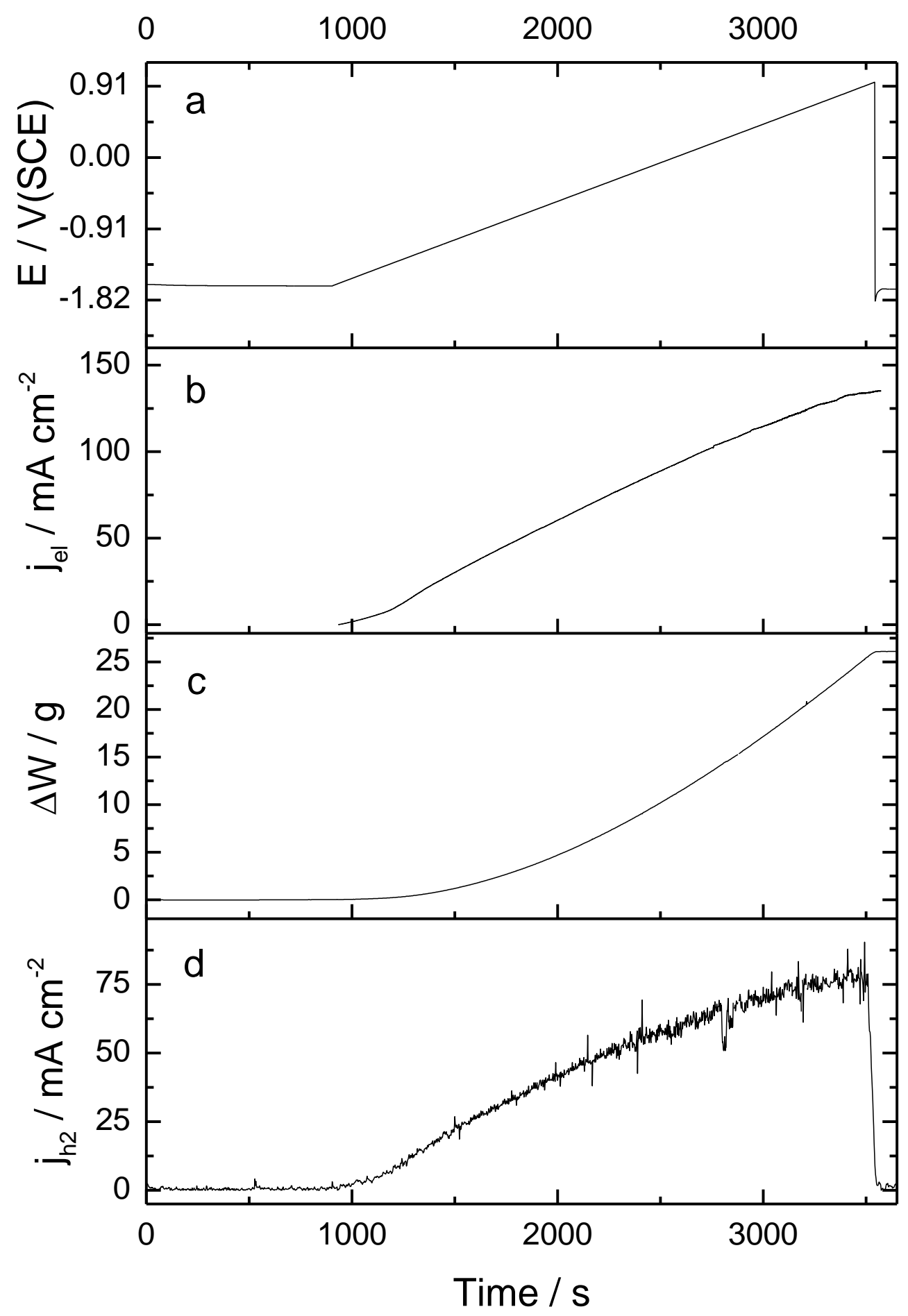

Figure 7. Time evolution of a) the potential, b) the electrical current density, c) the weight difference due to collection of the evolved hydrogen and d) current density associated to the evolved hydrogen. 


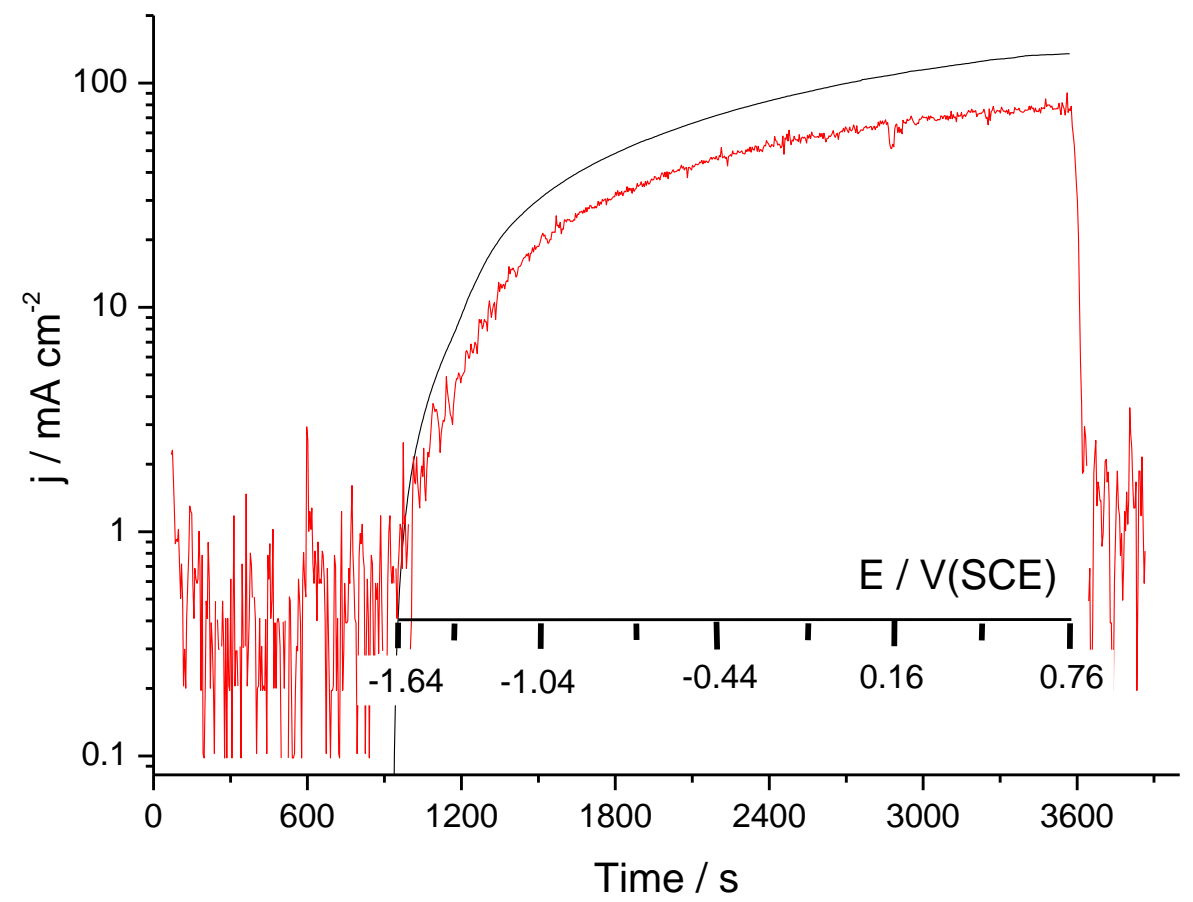

Figure 8. Comparison between the electrical current and the current associated to hydrogen evolution as a function of time and applied potential. 


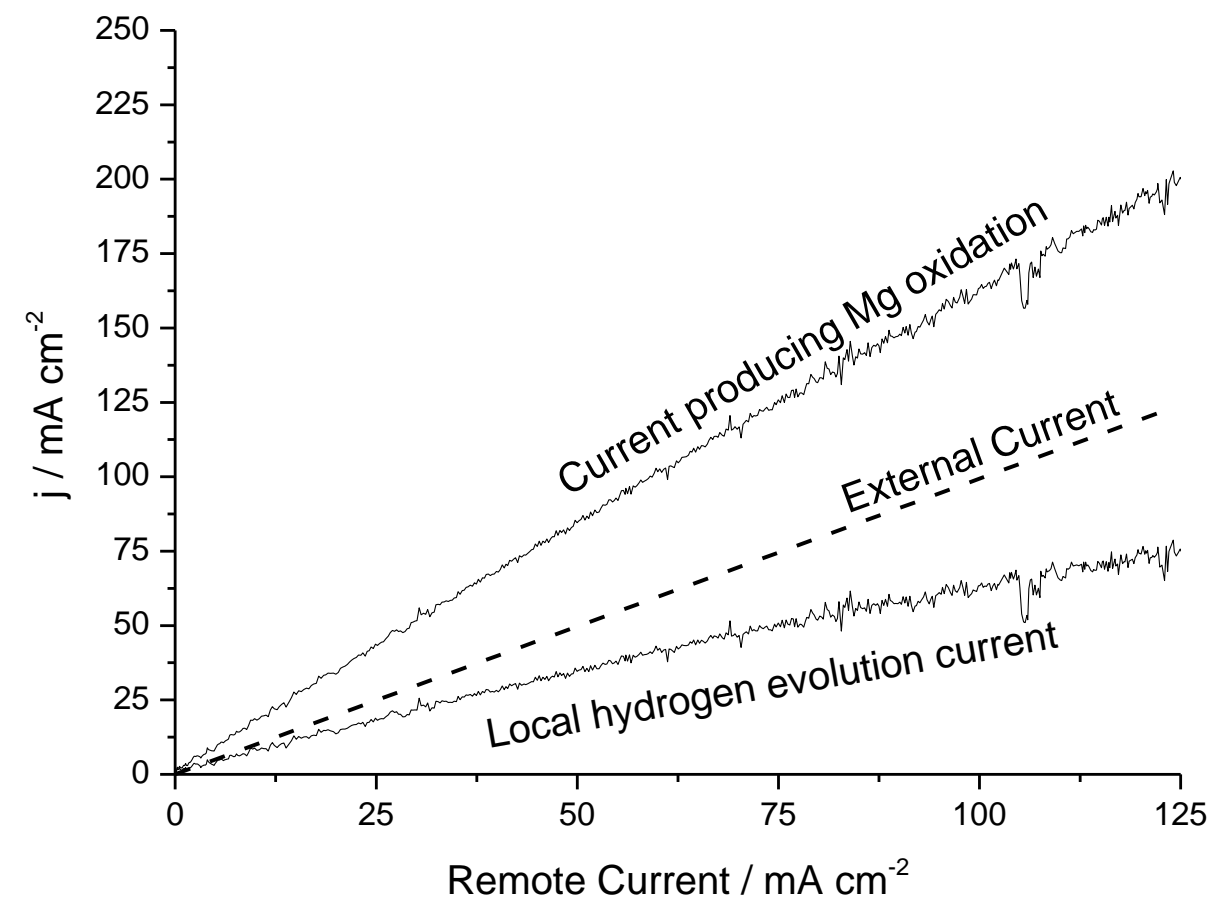

Figure 9. Dependency of the current producing magnesium oxidation on the value of remote current, under the assumption that during anodic polarization the external current largely exceeds the remote cathodic current generated on the electrode surface (ref. Figure 6). 